\title{
Design of Highly Nonlinear Bismuth-Oxide Holey Fibres with Zero Dispersion and Enhanced Brillouin Suppression
}

\author{
F. Poletti, P. Petropoulos, N. G. R. Broderick and D. J. Richardson \\ Optoelectronics Research Centre, University of Southampton, Southampton, SO17 1BJ, United Kingdom, \\ e-mail frap@orc.soton.ac.uk
}

\begin{abstract}
We demonstrate that $\mathrm{Bi}_{2} \mathrm{O}_{3}$-based holey fibres can achieve the same nonlinear performance as their allsolid counterparts, but with greatly improved dispersion control. Longitudinally tapering the structural parameters allows at least a further 13.5 times improvement in the Kerr-to-Brillouin figure-of-merit.
\end{abstract}

\section{Introduction}

A large number of devices based on the Kerr nonlinearity of optical fibres have been proposed and studied in recent years. For applications requiring the use of CW, narrow linewidth pumps, such as parametric amplification or FWM-based wavelength conversion, stimulated Brillouin scattering (SBS) is the main impairment, limiting the maximum amount of power that can be launched in practice into the fibre. In order to take the fibre's SBS limitation into account, a new Kerr-to-Brillouin figure of merit for highly nonlinear fibres has been proposed $\left(F O M=\gamma L_{\text {eff }} P_{\text {th }}\right.$, where $Y$ is the fibre nonlinearity, $L_{\text {eff }}$ is the effective length and $P_{\text {th }}$ is the SBS threshold) [1]. According to this definition it was demonstrated that solid core step-index fibres (SC-SIFs) fabricated using two different types of Bismuth-based glass provided a very high FOM due to the extremely high Kerr nonlinearity coefficient and the relatively low Brillouin gain coefficient of the core glass [1]. The large normal material dispersion of the glass at $1.55 \mu \mathrm{m}$, however, can only be partially compensated by the waveguide dispersion of SC-SIFs, and this is likely to limit the ultimate performance and use of these fibres.

It is well known that holey fibres (HFs), which can be made from a single glass, allow for far greater control of the overall dispersion. The fabrication technology for compound glass HFs has improved greatly recently and fibres with many holes, and complex geometries, can now be fabricated [2, 3].

In this study we investigate the design of HFs with hexagonally arranged holes made from the $\mathrm{Bi}_{2} \mathrm{O}_{3}$ based glass with 65.5 mol\% Bismuth concentration previously developed as the core material for SCSIFs. The key optical properties of this glass are presented in Refs. [1,4]. We propose a HF design that should provide a similarly large FOM to the solid SI version, but with zero dispersion and zero dispersion slope at $1.55 \mu \mathrm{m}$. Moreover, following the approach we recently proposed for silica fibres [5], we present tapering strategies that should allow at least a further 13.5 times increase in the FOM, while maintaining a very low average dispersion.

\section{Optical properties of Bismuth HFs}

We modelled the optical properties of hexagonally stacked $\mathrm{Bi}_{2} \mathrm{O}_{3} \mathrm{HFs}$ using a full vector finite element method, which allows the material dispersion to be directly accounted for. We focused on small-core HFs, and systematically modelled fibres with hole-tohole spacing $(\Lambda)$ between 0.5 and $1.5 \mu \mathrm{m}$ and relative hole size $(\mathrm{d} / \Lambda)$ between 0.6 and 0.8 . We found that all fibres within this parameter space are single mode and 4-5 rings of holes are sufficient to obtain a confinement loss that is negligible with respect to the bulk glass loss. We show the most relevant optical properties of $\mathrm{Bi}_{2} \mathrm{O}_{3} \mathrm{HFs}$ at $1.55 \mu \mathrm{m}$ in the map in Fig. 1 .

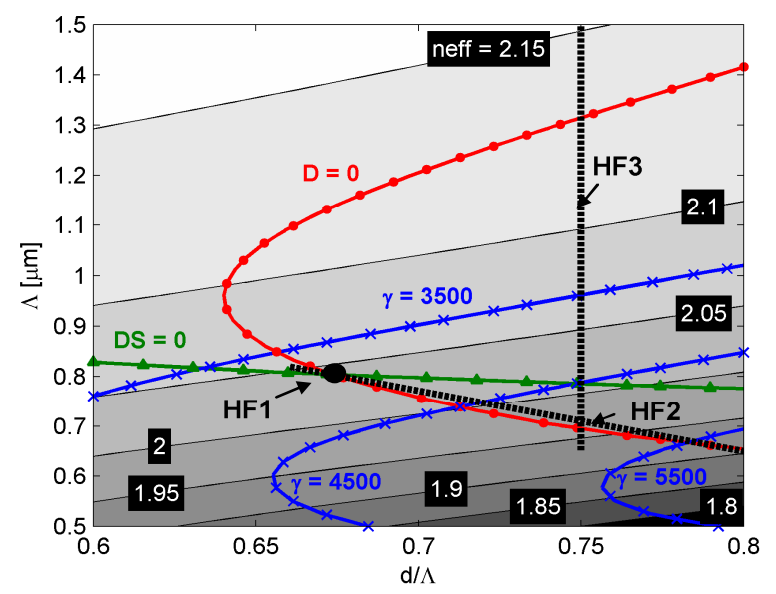

Fig. 1 Optical property map at $1.55 \mu \mathrm{m}$ for $\mathrm{Bi}_{2} \mathrm{O}_{3} \mathrm{HFs}$ showing the most relevant contour lines for effective index $\left(n_{\text {eff }}\right)$, dispersion $(D)$, dispersion slope (DS) and nonlinear coefficient $(\mathrm{V})$. 3 proposed HFs are also shown: HF1 is uniform, HF2 and HF3 are tapered.

This plot shows that fibre HF1 with $(\mathrm{d} / \Lambda, \Lambda)=(0.67$, $0.82 \mu \mathrm{m}$ ) has both zero dispersion and zero dispersion slope. HF1 has an effective area of $0.89 \mu \mathrm{m}^{2}, \sim 3$ times smaller than the SC-SIF version. The increase in nonlinearity ( $\mathrm{Y}$ ) would be accompanied, in the worst case, by a corresponding decrease in SBS thresholds. As a result, we would expect the HF and SC-SI versions to have a similarly high FOM ( 1). The simulated dispersion of the optimum fibre and the contour plots of the fundamental modes of both the SC-SIF and HF1 are shown in Fig. 2.

\section{Tapered HFs for improved SBS suppression}

A known method to broaden the overall Brillouin line is to apply a longitudinal change in the fibre structure in order to induce a nonuniform Brillouin Frequency 
Shift (BFS) along the fibre length. Since the BFS is to first order proportional to the effective index of the optical mode (BFS $=2 n_{\text {eff }} V_{a} / \lambda$ where $v_{a}$ is the velocity of the acoustic mode), and HF technology allows for a large range of $n_{\text {eff }}$ values (see Fig.1), we consider longitudinal structural tapering of HFs to be a particularly powerful means of increasing the SBS threshold for HFs [5]. Through accurate control of $(d / \wedge, \wedge)$ along the fibre length it is also possible to control the average value of dispersion, dispersion slope and nonlinearity.

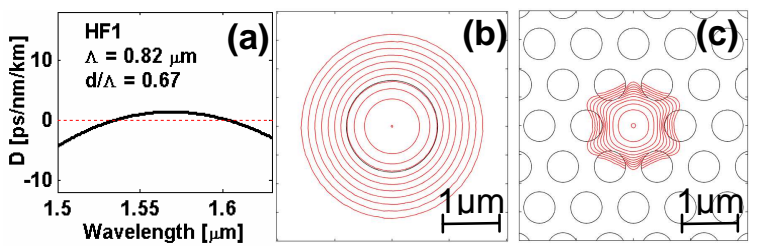

Fig.2 (a) simulated dispersion of HF1; $2 d B$ contour plots of the SC-SIF (b) and HF1 (c) at $1.55 \mu \mathrm{m}$

Our study shows that a tapered fibre (HF2) in which both $d$ and $\Lambda$ scale linearly from an initial $(d / \wedge, \wedge)$ dimension of $(0.66,0.82 \mu \mathrm{m})$ to a final value of $(0.8$, $0.65 \mu \mathrm{m}$ ) would closely follow the zero dispersion (ZD) contour line and have at the same time a large $\Delta n_{\text {eff }}$ and associated variation in BFS. Its main structural and optical parameters are shown in Fig. 3, where, due to the high value of nonlinearity, we assumed a tapering length of only $1 \mathrm{~m}$. In theory dispersion variation could be avoided altogether using a trajectory that follows the ZD contour.
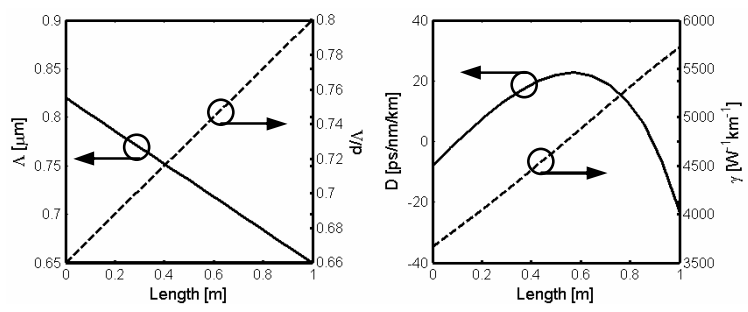

Fig.3 Structural and optical parameters of HF2

To establish the effect of the tapering on the fibre's Brillouin properties we follow the procedure described in Ref [6] and integrate the 'local' Brillouin gain curve along the fibre length in order to obtain an overall effective Brillouin gain curve. If we assume in the first instance that the detailed lineshape is not grossly affected by the microstructure, then we can use the measured BFS and Lorentzian Brillouin Gain Bandwidth (BGB) of $8.81 \mathrm{GHz}$ and $32 \mathrm{MHz}$ of the SCSIF fibre reported in Ref [1] to estimate the effective line shape and peak gain coefficient of HF2. By further assuming that through careful fabrication it will be possible to achieve similar losses for HF1 and HF2, as already obtained for SC-SIFs $(0.8 \mathrm{~dB} / \mathrm{m})$, we estimate that HF2 would have a Brillouin Gain FWHM of $\sim 600 \mathrm{MHz}$ (i.e. a 19x linewidth enlargement). This would provide an effective maximum gain coefficient suppression factor of 13.5, and a corresponding increase in the FOM. A comparison of the main properties of the SC-SIF, HF1 and HF2 is shown in Table 1 and 2.

Note that should the microstructuring itself result in more complex acousto-optic interactions in certain regions of the fibre design space, as is likely e.g. through interactions with additional higher-order acoustic modes, then this would broaden further both the effective local and overall Brillouin linewidths. Hence the FOMs presented here should be considered as likely lower bounds on the improvements that can be expected.

Table 1 - Comparison of optical properties

\begin{tabular}{|c|c|c|c|c|c|}
\hline & $n_{\text {eff }}$ & $\underset{[\mathrm{ps} / \mathrm{nm} / \mathrm{km}]}{\mathbf{D}}$ & $\underset{\left[\mathrm{ps} / \mathrm{nm}^{2} / \mathrm{km}\right]}{\text { DS }}$ & $\begin{array}{c}n_{2} \\
{\left[\mathrm{~m}^{2} \mathrm{~W}^{-1}\right]}\end{array}$ & $\underset{\left[W^{-1} \mathrm{~km}^{-1}\right]}{\mathbf{Y}}$ \\
\hline SC-SIF & 2.17 & $-260^{*}$ & 0.46 & & $1100^{*}$ \\
\hline HF1 & 2.05 & 0 & 0 & $8.17 \times 10^{-19 *}$ & 3720 \\
\hline HF2 & $2.05 \rightarrow 1.9$ & $8^{\#}$ & $0 \rightarrow-1.1$ & & $3660 \rightarrow 5730$ \\
\hline
\end{tabular}

Table 2 - Comparison of Brillouin properties

\begin{tabular}{|lcccc|}
\hline & $\begin{array}{l}\text { BFS } \\
{[\mathrm{GHz}]}\end{array}$ & $\begin{array}{c}\text { BGB FWHM } \\
{[\mathrm{MHz}]}\end{array}$ & $\begin{array}{c}\text { Brill. gain coeff. } \\
{\left[\mathrm{m} \mathrm{W}^{-1}\right]}\end{array}$ & FOM \\
\hline \hline SC-SIF & $8.81^{*}$ & $32^{*}$ & $6.43 \times 10^{-11 *}$ & 1.03 \\
HF1 & 8.32 & 32 & $6.43 \times 10^{-11}$ & 1.03 \\
HF2 & $8.32 \rightarrow 7.71$ & $606^{\#}$ & $4.76 \times 10^{-12}$ & 13.9 \\
\hline
\end{tabular}

\section{Conclusions}

We have performed a detailed numerical study of the nonlinear properties of hexagonally arranged HFs, fabricated with high $\mathrm{Bi}$ concentration $\mathrm{Bi}_{2} \mathrm{O}_{3}$ glass. We have identified a HF design that provides dispersion shifted and flattened properties for nonlinear applications within the C-band, and a high KerrBrillouin FOM. Moreover we have identified a linear tapering trajectory which, while remaining in a low dispersion regime, would allow a 19 times enlargement of the Brillouin linewidth and a further 13.5 times FOM improvement. Note that Fig.1 provides a convenient and very visual means to design fibres for other applications. For example, fibre HF3 $(0.75,1.5 \mu \mathrm{m}) \Rightarrow(0.75,0.65 \mu \mathrm{m})$, would present an effective BGB of $808 \mathrm{MHz}$, an average $\mathrm{y}$ of 3200 $W^{-1} \mathrm{~km}^{-1}$ and an average dispersion of $30 \mathrm{ps} / \mathrm{nm} / \mathrm{km}$, and would be of interest for low latency, increasedbandwidth, SBS based slow-light generation [7].

\section{References}

1 J. H. Lee et al, Opt. Letters, 30 (2005), 1698

2 J. Y. Y. Leong et al, ECOC 2005, PD Th 4.4.5

3 P. Falkenstein et al, Opt. Letters, 29 (2004), 1858

4 T. Hasegawa et al, OFC 2006, paper OTuH5

5 F. Poletti et al, OFC 2006, paper OWI10

6. K.Shiraki et al, J. Lightwave Technol. 14 (1996), 50

7. C. Jauregui et al, OFC 2006, paper PD1.2 